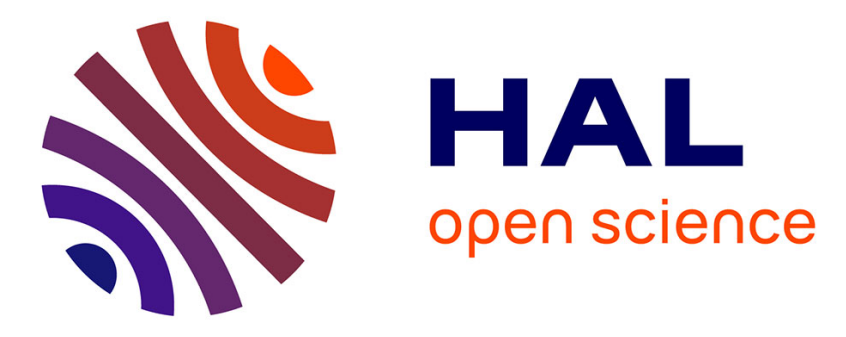

\title{
Introducing the scanning air puff tonometer for biological studies.
}

Vincent Fleury, Alia Al-Kilani, Olena P. Boryskina, Annemiek J. M.

Cornelissen, Thi-Hanh Nguyen, Mathieu Unbekandt, Loïc Leroy, Georges

Baffet, Ferdinand Le Noble, Olivier Sire, et al.

\section{To cite this version:}

Vincent Fleury, Alia Al-Kilani, Olena P. Boryskina, Annemiek J. M. Cornelissen, Thi-Hanh Nguyen, et al.. Introducing the scanning air puff tonometer for biological studies.. Physical Review E : Statistical, Nonlinear, and Soft Matter Physics, 2010, 81 (2), pp.021920. 10.1103/PhysRevE.81.021920 . hal00666187

\section{HAL Id: hal-00666187 \\ https://hal.science/hal-00666187}

Submitted on 11 Apr 2016

HAL is a multi-disciplinary open access archive for the deposit and dissemination of scientific research documents, whether they are published or not. The documents may come from teaching and research institutions in France or abroad, or from public or private research centers.
L'archive ouverte pluridisciplinaire HAL, est destinée au dépôt et à la diffusion de documents scientifiques de niveau recherche, publiés ou non, émanant des établissements d'enseignement et de recherche français ou étrangers, des laboratoires publics ou privés. 


\title{
Introducing the scanning air puff tonometer for biological studies
}

\author{
Vincent Fleury, ${ }^{1}$ Alia Al-Kilani, ${ }^{2}$ Olena P. Boryskina, ${ }^{3}$ Annemiek J. M. Cornelissen, ${ }^{1}$ Thi-Hanh Nguyen, ${ }^{4}$ \\ Mathieu Unbekandt, ${ }^{2}$ Loï Leroy, ${ }^{2}$ Georges Baffet, ${ }^{5}$ Ferdinand le Noble, ${ }^{6}$ Olivier Sire, ${ }^{7}$ Elodie Lahaye, ${ }^{7}$ and Vincent Burgaud ${ }^{2}$ \\ ${ }^{1}$ Laboratoire Matière et Systèmes Complexes (MSC), UMR 7057, Université Paris-Diderot, CNRS, Bâtiment Condorcet, \\ 10 Rue Alice Domon et Léonie Duquet, 75013 Paris, France \\ ${ }^{2}$ Groupe Matière Condensée et Matériaux, Institut de Physique de Rennes, Université de Rennes 1, CNRS, Campus de Beaulieu, \\ Bâtiment 11A, 35042 Rennes, France \\ ${ }^{3}$ Institute of Radiophysics and Electronics NAS of Ukraine, Acad. Proskura Str. 12, Kharkov 61085, Ukraine \\ ${ }^{4}$ Laboratoire de Physique de la Matière Condensée, Ecole Polytechnique, CNRS, 91128 Palaiseau Cedex, France \\ ${ }^{5}$ Laboratoire de Recherches sur les Pathologies Hépatiques, INSERM U 522, IFR 97 CHU, Hôpital Pontchaillou, \\ Université de Rennes 2-Villejean, 35033 Rennes, France \\ ${ }^{6}$ Laboratory for Angiogenesis and Cardiovascular Pathology, Max Delbrück Centrum für Molekulare Medizin, \\ Robert Rössle Strasse 10, 13125 Berlin-Buch, Germany \\ ${ }^{7}$ Laboratoire des Polymères, Propriétés aux Interfaces et Composites, Université de Bretagne-sud, Campus de Tohannic, \\ 56017 Vannes Cedex, France
}

(Received 28 August 2009; revised manuscript received 10 November 2009; published 22 February 2010)

\begin{abstract}
It is getting increasingly evident that physical properties such as elastoviscoplastic properties of living materials are quite important for the process of tissue development, including regulation of genetic pathways. Measuring such properties in vivo is a complicated and challenging task. In this paper, we present an instrument, a scanning air puff tonometer, which is able to map point by point the viscoelastic properties of flat or gently curved soft materials. This instrument is an improved version of the air puff tonometer used by optometrists, with important modifications. The instrument allows one to obtain a direct insight into gradients of material properties in vivo. The instrument capabilities are demonstrated on substances with known elastoviscoplastic properties and several biological objects. On the basis of the results obtained, the role of the gradients of elastoviscoplastic properties is outlined for the process of angiogenesis, limb development, bacterial colonies expansion, etc. which is important for bridging the gaps in the theory of the tissue development and highlighting new possibilities for tissue engineering, based on a clarification of the role of physical features in developing biological material.
\end{abstract}

DOI: 10.1103/PhysRevE.81.021920

PACS number(s): 87.85.G-, 83.85.-c, 83.60.-a, 87.80.Ek

\section{INTRODUCTION}

Measuring the mechanical properties of complex materials, such as embryonic tissues, is of utmost importance for understanding biological processes and regulations of genetic pathways [1]. Indeed, in addition to chemical gradients, the processes of morphogenesis (or pattern formation), remodeling, and regeneration are always characterized by the existence of mechanical gradients $[2,3]$. The critical role of the pattern of the mechanical force field has been put forward for the processes of growth and extension of new biological tissue, in situ differentiation of existing tissue with local variations of mechanical properties [4], e.g., mesoderm differentiating into oriented cartilage inside chondrogenic condensations [5], or extension of tracheal ducts in flies [6] and during any phenomenon which implies shear or strain, such as blood vessels remodeling under the action of blood flow [7]. However, the gradients of mechanical properties are often inferred indirectly by model-dependent mathematical analysis [6].

There exists a limited number of techniques capable of measuring the biomechanical properties of tissue in vivo. The most commonly used ones are optical tweezers techniques [8], cantilever techniques [9], magnetic beads techniques [10], particle tracking techniques [11], acoustic techniques [12], and also micropipette aspiration techniques [13]. All of the above techniques have their pros and cons. For example, acoustic techniques provide a rather poor spatial resolution, in the millimeter range. Optical tweezers techniques or magnetic beads imply inserting little beads in the tissue, which is not always easy, not even mentioning the disruption of local mechanical properties by the insertion. Particle tracking techniques are interesting for homogeneous materials, but not so much for very inhomogeneous materials. Cantilever techniques require a physical contact between the sample and the instrument, which renders point by point analysis difficult as the biological tissue have strong adhesion forces. Micropipette aspiration techniques are quite invasive.

Therefore, in order to match the demands in probing the mechanical properties of living samples in vivo, we have designed a new instrument, namely, a scanning air puff tonometer (SAPT), which is able to map point by point the elastoviscoplastic properties of flat or gently curved soft materials. The principle of the instrument is inspired by the air puff tonometer used by optometrists [14]. A fine air jet is used to indent the sample and the deformation is accurately measured optically. We present here after the detail of the instrumentation, and several case studies related to different scientific problems, in which the physical parameters of the biological situation may be crucial. Several technical details are given in the Appendix. The proposed instrument has three main limitations: (1) the air jet cannot be imparted for long on the surface (risk of desiccation), (2) the air jet probes 
only the outer layers of the tissue, and (3) it works better on flat surfaces. Still, homogenously curved surfaces (such as limb buds) can also be addressed. The most important conclusions derived from the set of experiments performed with the help of the SAPT and presented in this paper are as follows. The instrument allows performing high-precision measurements of the elastoviscoplastic parameters of different kinds of biological samples including bacteria colonies, yolk sac, liver tumors, limb buds, etc. The results obtained confirm the existence of compliance, or deformability, gradients in all studied living tissues, related to stress gradients, and growth directions.

\section{SCANNING AIR PUFF TONOMETER}

The principle of the instrument is the following: the sample surface is deformed by a focused air puff; the size of the deformation hole impacted on the surface is estimated optically and serves as a measure of the surface mechanical properties. In order to get valuable scientific information, the air puff must be localized, the deformation must be observed and measured accurately, and the air puff or the sample must be displaceable.

In order to complete this challenge, the instrument is constructed in the following way. The air puff is blown through a glass micropipette generated with a pipette puller (pip5 from Heka). The resolution of the instrument is in principle equal to the diameter of the pipette. However, mechanical forces are intrinsically long ranged, therefore, the geometry of the indentation is generally larger than the air jet itself (think of a book deforming a shelf). We present here below results obtained generally with tips in the $60 \mu \mathrm{m}$ range (we were able to generate functioning pipettes down to $10 \mu \mathrm{m}$ of diameter, results obtained with the help of these tips will be presented elsewhere).

The air puff is generated by a pressure line, controlled by an air port (PORTER Instruments, Ref. 8310), a pressure gauge (Kelatron), and a flux meter (GPE meterate 314). The pressure control included correction due to the atmospheric pressure variability since minute pressures (above $P_{\text {atm }}$ ), typically 5 millibar, are applied at the entry of the pressure line.

In order to accurately measure the deformation, a considerable attention was dedicated to the problem of observing and measuring the deformation during the air puff and the following setup was eventually designed. In this setup, the pipette itself is used as a guide for the spot of light, shown directly onto the deformation concavity. By so doing, the same tip emits almost coaxially an air puff and a light spot. It is absolutely crucial for reproducibility and unbiased data interpretation that the light spot, the air spot, and the optical detection be coplanar [Fig. 1(a)]. In addition, it is desirable that the air spot be oriented somewhat in the back of the light spot. By so doing, the travel of the light spot during deformation is one way and the signal-to-noise ratio is optimized [Fig. 1(b)]. However, the SAPT can also be used in a mode where the total dimension of the spot is used for deformation analysis (see the Appendix). Another possible setup for measuring the size and position of the deformation consists in

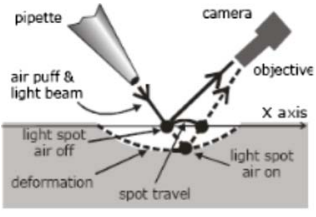

(a)

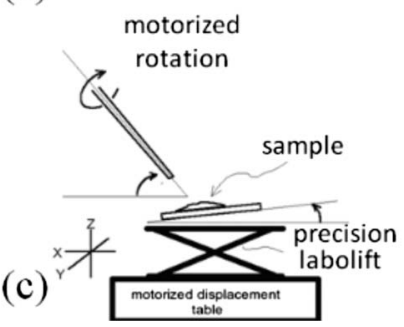

(d)

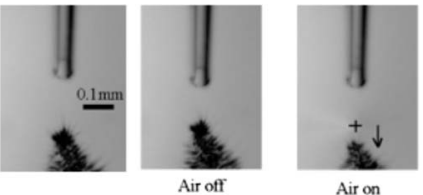

(b)

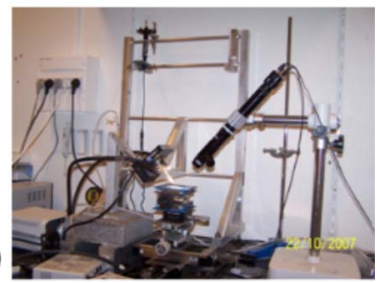

FIG. 1. (Color online) (a) Orientation of the pipette and the detection optics. In order to obtain a good signal-to-noise ratio when measuring the spot travel and the deformation, the camera and objective axes must be coplanar. (b) Actual images of tip and the sample surface (ethanol). Cross and arrow show the travel of the spot during the air puff. (c) In order to achieve the alignment of the tip and the positioning of the spot, the tip is motorized around its axis and the angle of the tip can be adjusted with a goniometer. The sample tilt angle can also be adjusted. (d) View of the entire setup (not included are computers and control screens).

observing the surface by the more classical shadowgraph technique [15].

Considering that each micropipette has its idiosyncrasy, it is necessary to place the micropipette on a rotative stage, in order to be able to turn the tip around its own axis, so that it can be properly oriented during installation of a new tip. The setup is equipped with the motorized rotative stage (Newport SNB070611). Another stage (Newport Gon65-U) serves for rocking of the tip in the plane (tip, spot, detector), which is important for positioning the light spot ahead of the air spot.

In order to generate the light spot, a cleaved and bared end of a multimode optical fiber "working fiber" (HCP MO200T from SEDI) is introduced inside the pipette. One end of the optical fiber is inserted as deep as possible inside the micropipette (Fig. 2). We also checked that the insertion was accomplished with no obstruction. The other end is coupled to the "injection fiber" using a splice device (Siemon ULTRAsplice U.S.-250). At last, a laser beam produced by a diode (12 $\mathrm{mW}$ Melles Griot) is injected in the "injection fiber" using an optic positioner FP-1A and a bare fiber chuck holder FPH-J.

The spot is observed at an angle of $90^{\circ}$ with an analog video camera (Watec 512) which continuously films the surface and records on line the position and size of the spot (with a frame grabber from SCION corporation interfaced with the free software NIHIMAGE created by Wayne Rasband). The surface is filmed through a microscope tube (Optem zoom70XL). A second microscope observes the sample perpendicularly, for proper checks and positioning, and also for the shadowgraph measurements. For the cancer data, a fluorescent microscope (Leica MZFLIII) was used for colocalization of the tumors (marked with a fluorescent dye) and of the deformation spot. 

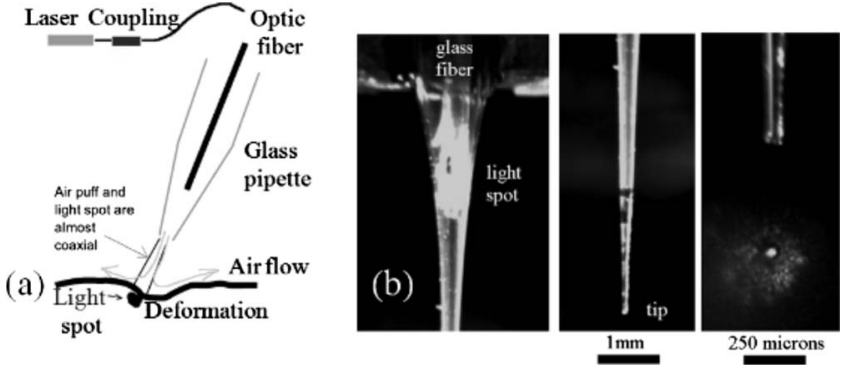

FIG. 2. (a) Schematic view of the pipette. A glass fiber is inserted inside the pipette and descended as far as possible toward the tip. Then the spot light and air puff are always close to be coaxial. The process of the pipette installation includes positioning of the pipette on the tonometer and rotation of the pipette until the air puff and light spot become coplanar with the optics. (b) A typical tip; the end of the inserted optical fiber, pipette tip, and tip apex with the final (outer) diameter of approximately $60 \mu \mathrm{m}$ are shown. The spot of light on the sample appears in the image to the right.

With our equipment, the accuracy of the spot position is of the order of 0.1 pixel on the monitoring screen, which is $0.8 \mu \mathrm{m}$ in metric units at magnification $\times 1$ of the microscope and $0.16 \mu \mathrm{m}$ at magnification $\times 5$, which was, for example, used for the blood vessel measurements. In addition, the samples are placed on a moving table (Newport ESP300) such that the surface can be scanned at speeds up to $0.2 \mathrm{~mm} / \mathrm{s}$ and with a maximal data-acquisition rate of $10 \mathrm{~Hz}$ (limited by the frame grabber, most data below were taken at $10 \mathrm{~Hz}$ ). The sample is also positioned on a precision labolift (Newport MEL80), so that the pipette-to-sample distance can be accurately fixed.

One typical run of a tonometry experiment consists of remaining at the same place, blowing air for a few seconds in order to generate an indentation of the surface, and recording the evolution of the surface during the entire air puff and during the recoil of the surface. Another typical run consists of scanning the surface at a constant air flow. This is only meaningful when the surface is very flat. A varied series of controls and examples is given in the Appendix.

\section{PHYSICS OF AN AIR JET IMPINGING ON A SURFACE}

Since the tonometer exerts a force on the surface, the deformed configuration probes the resulting reaction forces in the material. Three basic cases should be distinguished: elastic solid, viscous liquid, and viscoelastic material.

\section{A. Elastic solid}

In the case of an elastic material, there exists a constitutive equation relating the stresses and deformations (e.g., Hooke's law). At equilibrium, by Newton's law, the deformed configuration opposes itself to the force exerted by the air jet (elastic materials). The former appears in the boundary condition of the solid and continuity of the stresses at the boundary closes mathematically the problem (fluidstructure interaction). In this case, the tonometer probes the elastic parameters (spring analogy).

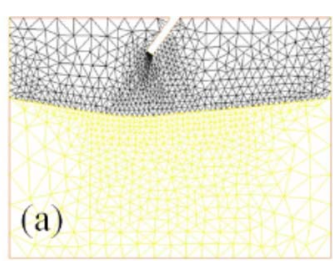

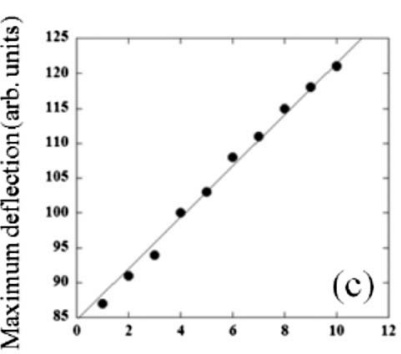

Airflux (arb. units) (b)
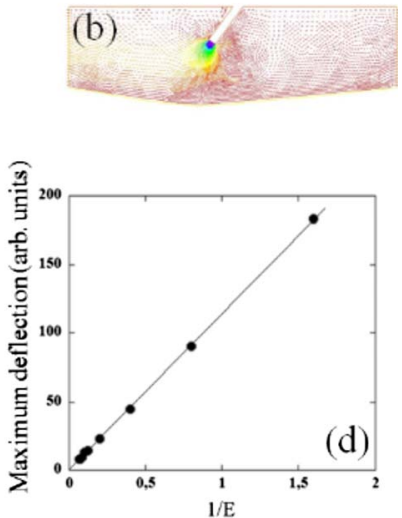

(d)
FIG. 3. (Color online) (a) Finite element mesh for the calculation of the deformation inside a solid exposed to an air puff. Black mesh (top mesh): the air, yellow mesh (bottom mesh): the solid. (b) Calculation of the Navier-Stokes solution for the air puff coming out of a pipette. The calculation was performed with the FREEFEM ++ code of the INRIA (courtesy of Pironeau and Hecht) with fluidstructure interaction conditions. (c) Calculated deflection as a function of the air flux at the pipette end. (d) maximal deflection of the solid, as a function of the parameter $1 / E$, with fixed air flux and fixed Poisson ratio. The numerical codes of the finite elements method (FEM) calculation are in arithmetical dimensionless units. The scaling parameters are the absolute dimension (linear scale unit) of the calculation. The density and the viscosity of the air can be fixed arbitrarily, the air speed at the exit of the pipette can be fixed arbitrarily, and the elastic parameters of the surface (Poisson ratio and Young modulus) can be fixed arbitrarily.

To be more specific, for a solid, we need to solve the deformation of the solid under the air jet. We have written a FREEFEM ++ finite element code for calculation in two dimensions (2D) of the deformation of an elastic solid with arbitrary values of elastic constants under a constant air jet coming out of a pipette, which can be provided upon request (Fig. 3). The calculations show that the behavior of the response is linear for a large range of parameters (air flux, elastic constant), which is quite suitable for our purpose which is to measure material properties under a gentle and short air puff.

\section{B. Surface of a liquid}

A liquid may deform statically in a way similar to a solid if it possesses a surface. In this case, the surface has a trivial constitutive equation by which the surface tension is a material constant, say $\sigma$. Then, a statically deformed fluid surface exerts a stationary force solely related to the static surface deformation (membrane analogy).

In more detail, the problem of an air puff impinging on a liquid surface was studied by Labus and Aydelott [16]. These authors showed that the vertical deformation is inversely proportional to surface tension in a range of parameters, controlled by the tip-to-surface distance, which we note $H$ and 
the pipette diameter noted $d$. Indeed, there exists actually a limitation related to viscous dissipation of the jet in the surrounding air before hitting the surface and also a secondorder effect of gravity. These authors introduce the Bond number Bo, and the Weber number, We, in order to take into account the effect of gravity, surface tension, and inertia

$$
\mathrm{Bo}=\rho_{1} g d^{2} / \sigma
$$

in which $\rho_{1}$ is the density of the liquid upon which the air jet impinges (say water or ethanol in our controls), $g$ is the acceleration due to gravity, and $\sigma$ the surface tension of the liquid. This number compares the respective roles of gravity and surface tension. Now, the Weber number reads

$$
\mathrm{We}=\rho_{\mathrm{gas}} V_{\mathrm{gas}}^{2} d / \sigma,
$$

in which $\rho_{\text {gas }}$ is the density of the impinging gas (here, air), $V_{\text {gas }}$ is the average speed of the gas, and $\sigma$ the surface tension of the liquid. This number compares the effects of surface tension and inertia.

The Reynolds number relates the effects of viscosity (noted $\nu_{\text {gas }}$ ) and that of inertia

$$
\operatorname{Re}_{\text {jet }}=\rho_{\text {gas }} V_{\text {gas }} d / \nu_{\text {gas }} .
$$

In our case,

$$
\begin{gathered}
d=50 \mu \mathrm{m}, \\
g=9.81 \mathrm{~m} \mathrm{~s}^{-2} .
\end{gathered}
$$

Tabus and Aydelott show that the maximal deformation $w$ of the surface (center of concavity) takes the form

$$
w=d \mathrm{We} /\left(K_{1}+K_{2} \mathrm{Bo}\right)
$$

and $K_{1}$ and $K_{2}$ are scaling parameters which depend on the pipette-to-surface distance $H$ and the diameter of the pipette $d$. These parameters are constant when the ratio $H / d<3$.

In our setup and experimental conditions, the air speed is of the order of meters per second, the pipette-to-surface distance is of the order of 50-200 $\mu \mathrm{m}$, and the pipette diameter is of order $50 \mu \mathrm{m}$. Therefore, from the analysis of Tabus and Aydelott, the SAPT will measure surface tensions, although on the upper boundary of the scaling law. (Bo $\sim 3$ $\times 10^{-4}$ for water, $\sim 8 \times 10^{-4}$ for ethanol.) We calibrated the tonometer on such liquid surfaces as water and ethanol (see the Appendix) in order to check the linearity of the deformation as a function of air flux and to check the effects of varying $H$ and varying $\sigma$.

\section{Viscoelastic behavior}

Many materials have a complex viscoelastic behavior that can be modeled by a combination of dashpots and springs. In the simplest case, a viscoelastic behavior can be described by a single spring and a single dashpot in series (Maxwell equation [17]) or in parallel (Kelvin-Voigt equation [17]). More complex cases are described by more complex combinations. In the first case, there is a rapid elastic response followed by a material flow. In the second case, the material reaches eventually an equilibrium after a viscoelastic transient. In

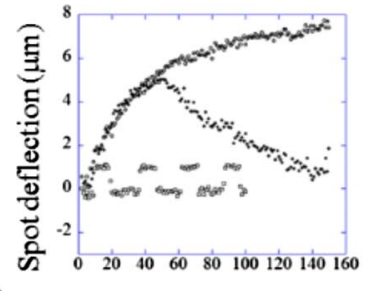

(a)

Time(0.1sec)

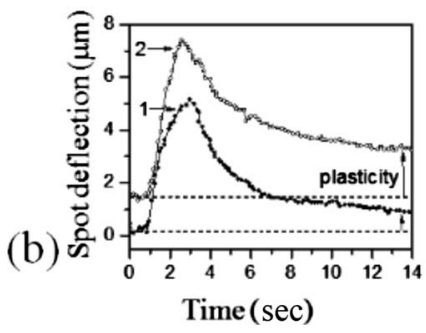

(c)

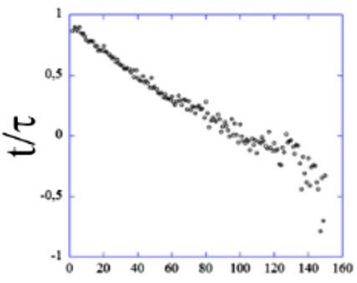

Time (0.1 sec)

FIG. 4. (Color online) [(a) and (c)] Analysis of the viscous behavior during the viscoelastic response of the gel as compared to the elastic response of a surface of water (large circles forming a crenel below the gel data). A constant air puff is imposed onto the surface for, say $15 \mathrm{~s}$, and the amplitude of the deformation is analyzed [in (a) right] by plotting $\{\log [\operatorname{deflection}(\infty)$-deflection $(t)\}$. We find a conspicuous straight line, therefore, the gel has a simple viscoelastic behavior in this range of force associated to a single relaxation time $\tau=\nu / E$. (b) Comparison of the reaction of a $2 \%$ agar preparation in a Petri dish to an air puff at 3 millibar (curve 1) and 9 millibar (curve 2). An irreversible deformation is generated, which can be seen directly on the surface by shadowgraph from the top [(c) left] or from the view at $90^{\circ}$ [(c) right, the tip was moved somewhat away from the crater, the light emitted by the spot allows one to see the crater). This irreversible deformation is due to yield stress of the gel.

this case, the final equilibrium corresponds to the elastic equilibrium of the spring (no deformation rate, eventually).

We analyzed a viscoelastic material, namely, the agar gel used as substrate for bacteria growth in the sequell (see Sec. IV A). Figure 4(a) shows the reaction of a $2 \%$ agar solution to a small air puff. The pattern of deformation is reproducible and reversible (the peak in the curve underneath corresponds to the moment when the air is turned off and the surface is left to relax). In this case, the analysis of the deformation shows a simple relaxation time of the viscoelastic material, associated to a Kelvin-Voigt-type behavior: after an exponential transient, the system reaches a saturated deformation. If the air stress is stopped, the system recoils to the initial position after a viscous transient. There is a single relaxation time $\tau$ equal to $\nu E$, where $E$ is the spring stiffness and $\nu$ the dashpot viscosity.

However, for larger puffs such as the two air puffs in Fig. 4(b), there remains a small irreversible indentation in the form of a crater [Fig. 4(c)], which is ascribed to a nonlinear yield stress. In the absence of a deeper analysis of the nonlinearities of the air-surface interaction, care must therefore be taken in order to remain in a simple viscoelastic regime. Extension of the instrument to nonlinear studies will be studied in the future.

Most biological samples studied (the results are shown further in this paper, Sec. IV) were found to behave almost 
elastically within the range of this study. They exhibited a limiting deformation after a rapid transient. This is also true for liquid surfaces.

In order to assess the viscoelastic relaxation time of the agar gel during the viscoelastic response, the air puff is maintained for as long as possible and the data following the initial transient are analyzed assuming a simple viscoelastic behavior of the form $A_{0}(1-\exp (-t / \tau))$. Figure 4(a) left shows an air puff over agar $2 \%$ maintained for $15 \mathrm{~s}$ (black dots) and an analogous gel reaction at a different spot of the same Petri dish (open circles) for an air puff (15 millibar) which is interrupted at $5 \mathrm{~s}$ : data are clearly reproducible. In the bottom of the graph, the crenel data correspond to the calibration on a water surface (four air puffs) in the same conditions (same tip, same air pressure). During a constant air puff, the strain of the viscoelastic material increases monotonously. If the air puff is interrupted, a damped recoil is obtained. Data of the continuous air puff were analyzed by subtracting the last value, changing sign, and plotting the logarithm of the amplitude of the deflection. For a linear viscoelastic behavior, a simple straight line with a single relaxation time is expected and indeed obtained in Fig. 4(a), right, except for very short and very long times (elastic domain and nonlinear creep at large deformations), with $\tau$ $=6 \mathrm{~s}$.

An anonymous reviewer has pointed our attention to an article introducing an instrument somewhat similar to this one [18]. In order to test the acoustic response of individual hair cells of the cochlea, the authors have used a fine pipette filled with a fluid and approached very close to the cell under study, itself fixed. The fluid in the pipette is excited by an acoustic vibration which propagates to the tip and next, the acoustic wave propagates to the cell through the air. This setup is superior to ours in terms of oscillatory stimulation, but was apparently not used for spatial analysis in vivo. It would be interesting in the future to use such an oscillating tip to assess the full range of viscoelastic response, spatially. However, while the response to the acoustic oscillatory vibration (no mean force) is of utmost importance for hearing, in the case of biological morphogenesis, the cell behavior (chronic push-pull action) is conceptually closer to the air puff action (chronic force).

\section{BIOLOGICAL EXAMPLES}

We now turn to biological examples. The samples are presented in increasing technical difficulty, although they were not studied in this order.

\section{A. Case study 1. Bacteria colonies}

Bacteria colonies of Proteus mirabilis expand by forming successive rings characterized by the alternation of two bacterial phenotypes: swarming and vegetative or "quiescent" [19]. The origin of these rings is not well understood. It has been suspected that it is related to a switch between different phenotypes of the bacteria itself depending on the physical properties of the substrate. The coupling between phenotypes and physical properties is in turn related to bacteria invasive-
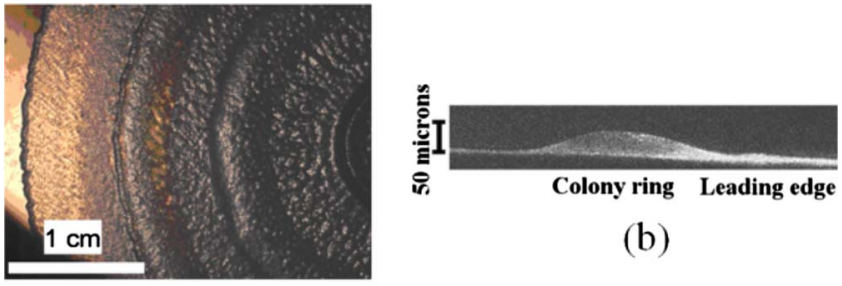

(b)

(a)

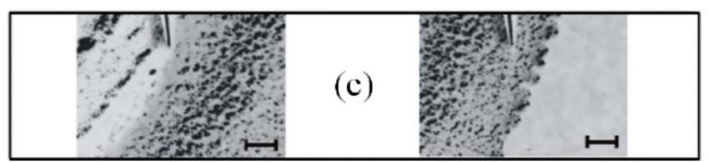

FIG. 5. (Color online) Shadowgraph and fluorescent confocal images of Proteus mirabilis colony on 2\% agar medium. (a) General view of approximately one half of the colony with the rings of expansion (shadowgraph). The radius of the colony is $3 \mathrm{~cm}$. (b) Cross section of the colony across one of the rings (staining by SYTO9/propidium iodide $5 \mu \mathrm{l}$ shed directly over the colony, confocal microscopy of the green fluorescence with a Zeiss microscope). (c) (Left) Local relief in internal area of the colony seen by shadowgraph. The tip of the pipette is seen; the size of the bar is $200 \mu \mathrm{m}$. (c) Right. Local relief at the edge of the colony (agar substrate to the right). The tip of the SAPT pipette is visible in the upper part of the image; the size of the bar is $200 \mu \mathrm{m}$.

ness as ring expansion will depend on bacteria motility. Actually, the behavior of the bacteria seems to be dependent on the stiffness of the substrate, which they progressively modify, by forming their biofilm. It is therefore believed that these rings are associated to different stiffnesses or viscosities (gradient of water activity will be modified during a swarming phase) induced by production of surfactants and polysaccharides [20,21]). These contrasts of viscoelasticity induce different motilities of the cells and hence of the bacteria invasiveness, therefore it is important to measure accurately the local viscoelastic properties. Current models ascribe the switch to a quiescent phenotype to a more fluid medium (Refs. [20,21]). We therefore analyzed by SAPT a bacteria colony (see sample preparation in Sec. VI A).

Figure 5(a) shows a shadowgraph image of the bacteria colony cultured on $2 \%$ agar, showing the different rings, corresponding to different mechanical parameters, already visible as the surface has a different roughness and swelling features. We analyzed by SAPT the boundary of the colony-where bacterial motility is maximal - and a more internal area of the colony-where no motility is observed. We performed this analysis by following the deflection of the spot and also by measuring directly by shadowgraph the diameter of the indentation. Figures 5(b) and 5(c) give the local images of the areas which were investigated. Figure 6(a) gives the direct integration of the deformation diameter during the air puff and Fig. 6(b) gives the result of the tonometer. The shadowgraph data are less noisy because it integrates over the entire indentation, while the SAPT spot images also the nonlinearities of the inside of the deformation cuvette. However, the same trend is observed, namely, that the internal area of the colony is more fluid than the boundary, such that the quiescent phenotype is indeed related 

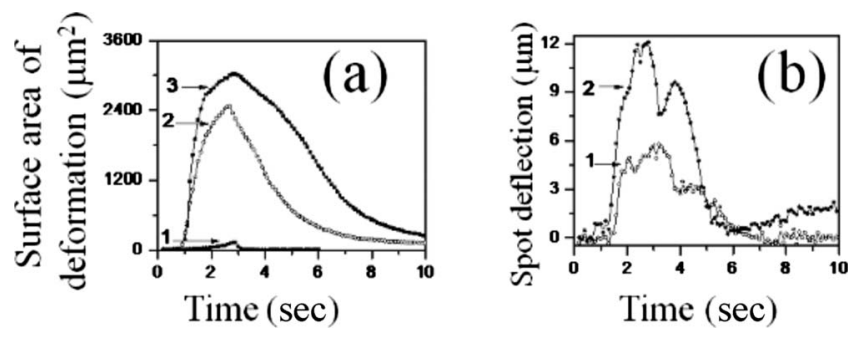

FIG. 6. (a) Deformation measured by direct integration of the deformation surface, as seen by shadowgraph, where curves 1, 2, and 3 correspond to pure $2 \%$ agar medium, area in the central part of the colony, and area at the edge of the colony, correspondently. (b) Same measurements performed by measuring the spot deflection where curve 1 is obtained in the central part of the colony and curve 2 at the edge of the colony. The features in the curves (b) correspond to actual nonlinearities of the displacement inside the deformation concavity due to the actual shape of the concavity (not parabolic). These details do not appear in (a), which integrates over the entire deformation. However, the general trend is the same. Both measurements find a gradient of compliance, the inside being more compliant than the very edge. Please note that the two techniques do not sense the same thing: the shadowgraph (a) senses the surface area of the hole, while the SAPT (b) senses the depth of the hole in the area of the light spot.

to a more fluid colony as already evidenced (Ref. [21]). The boundary was also compared to the agar just outside and a very large compliance difference was found.

\section{B. Case study 2: Formation of veins parallel to arteries}

There is increasing evidence that blood vessels are patterned by physical stresses in the embryo. However, while it has long been admitted that flow inside the vessels participates in vascular formation $[7,22,23]$, it was only recently realized that stress exerted by the surrounding tissue also is able to act as a "morphogene" on vessels $[15,24]$. Chronic viscoelastic deformations shape progressively the tissue, including its vessels. This chronic and progressive remodeling leads eventually to massive reorganizations of the vessels which can hardly be ascribed to a single genetic or biochemical cause. Especially, during formation of blood vessels, it is an obvious observation that veins tend to form somewhat parallel to arteries, but at "some distance" and that capillaries in between are rarefied. This is observed in the yolk sac, but also in other organs, such as the brain [Fig. 7(a)], the human placenta [Fig. 7(b)], and the mesentery or the retina (personal observation, data not shown). Figure 7(c) shows a chicken yolk sac at 5 days of development. At early stages, veins and arteries share the same vascular field (the so-called "plexus"), with arteries and veins located "in series." But progressively, a deterministic parallel pairing of vessels appears. This is well known in anatomy, as, for example, the so-called "venosa concomitans" as described in the arms and legs [see Ref. [25] and references therein] and also evident, for example, in the placenta, where arteries and veins form the umbilical chord, but also paired vessels in the placenta itself radiating away in all directions from the umbilical chord [Fig. 7(b)]. In addition, veins and arteries may often be found in different geometrical plateaus, the arterial plateau being located underneath the venous plateau, in the yolk sac. In the chorio allantoic membrane (CAM), in the retina or in the brain, veins will tend to be more "outwards" and arteries more "inwards" with respect to the geometrical center of the organ (data not shown). These physical features, which appear dynamically (typically in 1-2 days after beginning of perfusion in a yolk sac), require an understanding of the arterovenous interaction, viewed as a dynamic process which progressively shapes both of them locally and globally. It is important to understand the dynamics of this interaction because the formation of veins parallel to arteries is associated to a progressive disconnection of the capillaries located in between. While this disconnection is instrumental in allowing the formation of pairs of arteries and veins, it may induce pathologies if there is an excess of disconnection because in this case, the process ends in arteries which hardly perfuse the tissue [24]. This is especially obvious in the brain where avascular regions around arteries are called Virchow's spaces and have been related to dementia [26] but also in other parts of the body, where capillary rarefaction may occur as a consequence of pathological conditions, as, for example, in diabetes [27].

Regarding this arterovenous dynamic interaction, one observes at early stages of yolk sac development that while capillaries shrink along the arteries, they start to enlarge at some distance from the existing arteries [Fig. 7(c) and 7(d)], suggesting a direct physical or chemical induction of veins by the existing arteries, which are always first to appear, in the yolk sac. Direct observation of the early arterioles shows the presence of dilated cells, regularly stacked along the vessel wall [Figs. $7(\mathrm{e})-7(\mathrm{~g})]$. These cells are so dilated $(\sim 15 \mu \mathrm{m}$ in width) that they swell the surroundings of the arteriole [Figs. 7(h) and 7(i)], suggesting actually a physical (mechanical) interaction explaining the crushing of capillaries and the repulsion of the venous bed. This situation would naturally explain the "multifactorial" essence of several pathologies which end in capillary rarefaction: disruption of osmotic balances of different nature would have eventually a similar effect (crushing of capillaries).

In order to relate this phenomenon to physical properties of the tissue, we therefore analyzed in vivo the mechanical properties of the neighborhood of very young arteries by SAPT and evidence that the neighborhood of arteries, in the swollen region, is indeed much stiffer than the region located further away, in-between arteries [Fig. 7(j)] at this early developmental stage. Therefore, it is natural that the return flow will spontaneously flow away from arteries by a poroelastic effect and that a biomechanical feedback, namely, increase of diameter in the presence of an increased flow, will progressively generate a true blood vessel exactly parallel to the arteries, but outside the stiff area [25], in which capillary rarefaction is observed. In some sense, the stiffening of the area around arteries, linked to turgor pressure of the cells, expels the flow and creates an "exclusion" area between arteries and the forming veins, thus ensuring that there is no direct shunt between arteries and veins at this early stage.

This phenomenon has several remarkable consequences. One consequence is that arteries will tend systematically to have one or even two veins exactly parallel to themselves, as 


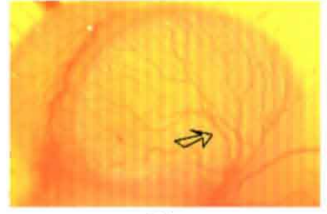

Oh

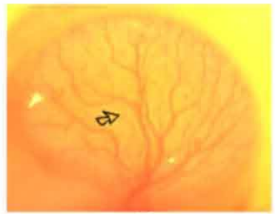

$8 \mathrm{~h}$

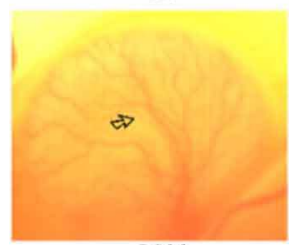

$23 \mathrm{H}$

(a)

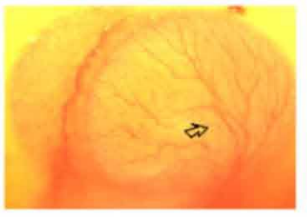

5h30

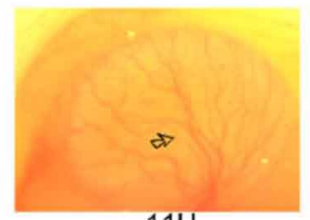

$11 \mathrm{H}$

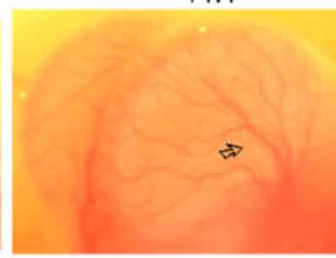

29h

$1.5 \mathrm{~mm}$
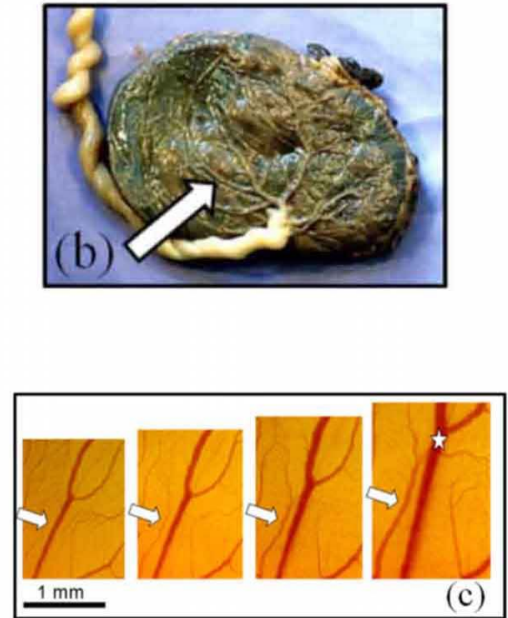

(c)
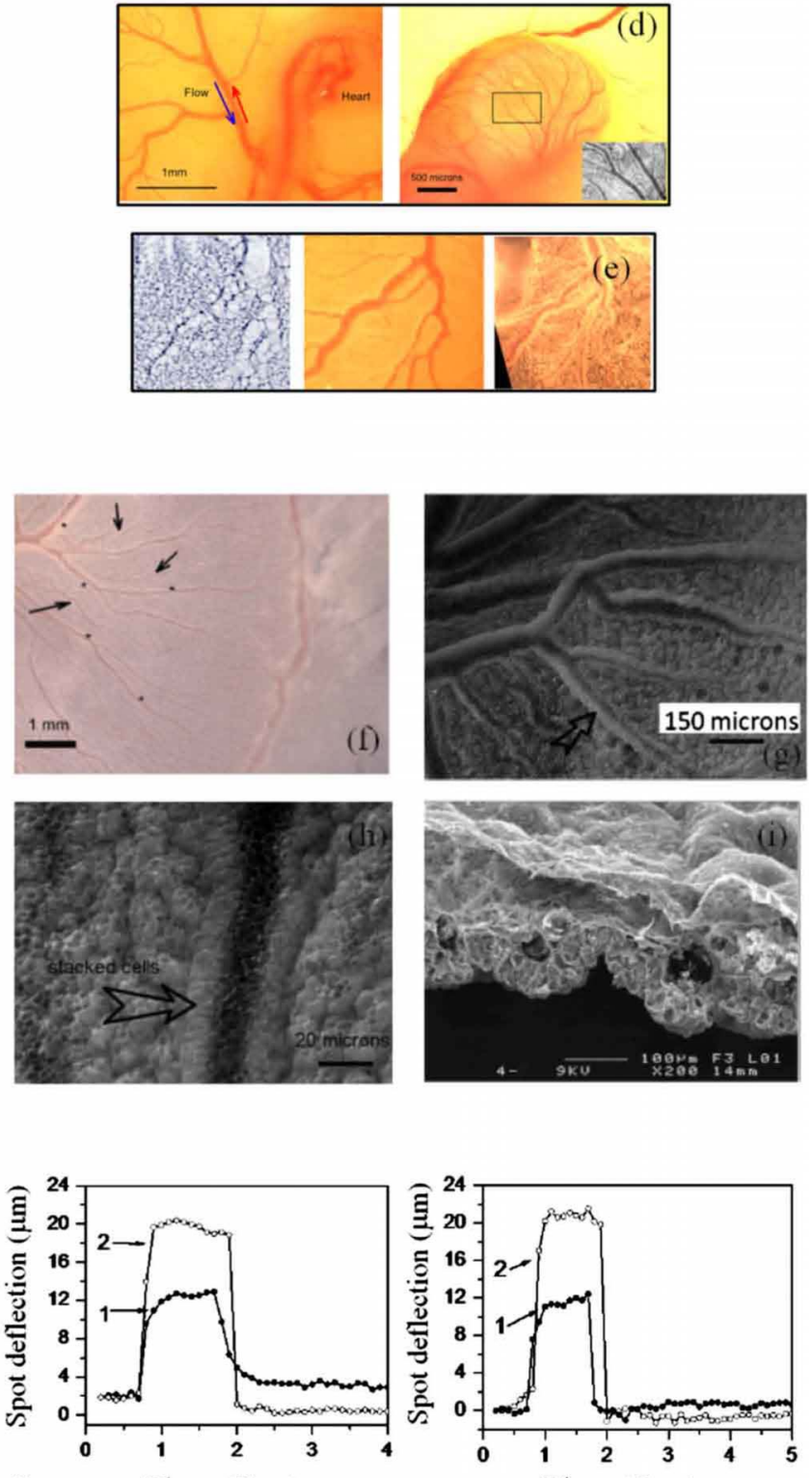

(j)

Time (Sec.)

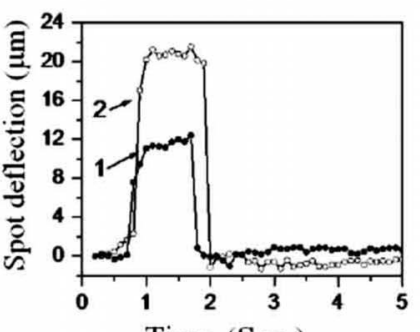

Time (Sec.)

FIG. 7. (Color online) (a) Sequence of chicken embryo brain development (telencephalon between 7 and 8 days) showing the formation of veins paralleling arteries. These images were acquired in vivo (see materials and methods). (b) Fresh human placenta showing the radiating paired vessels, which emanate from the umbilical cord (from Centre Hospitalier Universitaire Paris Jussieu). (c) At 4 days of development, in the chicken yolk sac, arteries already exist, while parallel veins start only to form (arrows). The sequence of images shows a $1 \mathrm{~h}$ interval in vivo time-lapse of vascular reorganization. In a few hours, the capillaries adjacent to an artery remodel into a vein parallel to the said artery. The artery is separated from its surrounding capillaries, as it is obvious from the fact that veins continue to mature and can even "cross" the existing artery (star). In fact, the vein does not pass "through" the artery, it follows the capillary path, disconnected from the artery, and crosses it from atop. (d) shows a typical vascular bed at day 5 of development in the yolk sac and at day 7 in the brain: arteries and veins are already closely parallel in the yolk sac. In the brain, capillaries gather on each side of an artery to form two venous paths. (e) (Left) Shadowgraph imaging of the topography shows that there is a swelling of the area surrounding the vessel, associated to the endoderm shell around the vessel. In (e) right, the endoderm image is taken from underneath on a rinsed yolk sac. It shows the shell of cells surrounding the vessel (these cells appear as a whiter area). The shell of endodermal cells does not show up clearly on the direct optical inspection of the same sample [(e) middle]. (f) Veins tend to form parallel to the existing arteries, but there exists an area with fewer capillaries around the arteries (visible in white, by absence of erythrocytes). In this area, the cells are very large cuboidal cells (10-15 $\mu \mathrm{m}$ in length) stacked in an organized fashion, orthogonal to the vessel direction, and in an orthoradial order [(g) and (h)]. The swollen area is pointed by the arrow in $(\mathrm{g})$ and $(\mathrm{h})$. Scanning electron microscopy shows that these cells contain very large droplets of fatty acids which saturate the volume of the cell (i). We analyzed by SAPT the swollen area region around arteries [(j), two examples]. The SAPT reveals a much stiffer area close to arteries (curves 1), in the swollen area, as compared to the area away from the artery (curves 2) (approximately a factor of 2 of compliance away from the artery). 
a consequence of capillary remodeling on each side (see in Fig. 7, the case of the brain; Ref. [25] contains also examples in the yolk sac), while the area in between arteries and veins will contain few capillaries. This is a consequence, on one hand, of the snow-ball effect of capillary enlargement under shear and, on the other hand, of the quasi-two-dimensional nature of biological tissues or sheets, such that (depending possibly on other boundary conditions) the physical stiffening of the surroundings of the artery creates two favored paths at the boundaries of this stiff area on both sides of the artery.

The origin of the excess of stiffness close to the arteries seems readily to be associated to the physical swelling of cells close to the arteries, which is very conspicuous, and explains naturally the capillary crushing. These cells are very large, about 3 times as large as the other cells present in the yolk sac, and they form a more regular pavement than cells further away from the vessel. These cells are in continuity with the underlying layer of endoderm. The endoderm is the cell layer located in contact with the yolk and its role is to take up the yolk, metabolize it, and transfer it to the vessels (and of course eventually blood flow brings it to the embryo). It is therefore normal that these cells be swollen with the fatty acids pumped from the underlying yolk, as confirmed by scanning electron microscopy [Fig. 7(i)].

However, if direct uptake of fatty acids from the yolk were the explanation of cell swelling, this mechanism would not exist in brains, CAMs, retinas, etc. We therefore performed an experimental assay, in which the embryo is entirely removed from the yolk sac, rinsed, and turned upside down. Then, yolk is added directly onto one half of the yolk sac only, the other side serving as contralateral control. The embryo survives and can be cultured this way in standard incubators. The result of this experiment shows that in this case, the vessels which are not in contact with the yolk undergo a massive dilation, which is extremely conspicuous, even spectacular (see Appendix, Sec. 6). This suggests that it is not directly the fatty acids of the yolk that regulate the swelling of cells and that this cellular swelling is more general than just the yolk-sac case.

In addition to being larger, these cells have distinct topological properties which change progressively. They have a more random distribution at very early stages and get progressively more organized in a conspicuous stacking order, orthogonal to the vascular direction, and with an orthoradial alignment of cells, as the artery becomes bigger. In addition, when cells are larger in the assay, the diameter of the vessels is also larger. The rationale therefore would be that increased circumferential stretch reorients and aligns physically the cells located along the arteries. These cells would swell as a consequence of physical tension which would allow a higher influx of fatty acids and/or other chemicals participating in cell physiology (salts, etc.). Whatever its cause, the swelling of the cells will tend to squeeze and disconnect the surrounding capillaries. Hence, veins will form somewhat away from arteries, outside this more ordered, swollen, area, in which capillaries are squeezed. Of course, this swelling may be an important issue in pathologies if there is an excess of it.

Also, since these swollen endodermal cells, which wrap the arteries, progress from underneath upwards, the arteries get disconnected preferentially in the deeper endodermal area first and progressively more toward the top layer, thus explaining naturally the observed segregation of veins and arteries into two plateaus, veins forming more "on top" of arteries which will be found underneath the veins. A more elaborated theoretical analysis of the problem of artery and vein pairing has been published recently in a specific paper [25], in which more extensive tonometry measurements are shown. Further work on this problem will consist in measuring the orientation order of cells (order parameter) along vessels as a function of tension in the vessel wall and measuring the respective viscoelastic properties of endothelial cells vs endodermal cells, since the competition between these two may be one factor regulating capillary disconnection.

\section{Case study 3: Liver tumors}

It was proposed recently that the mechanical properties of tumors play a role in their invasiveness and overall biochemical behavior [28]. We therefore compared the compliance of tumor and sane tissue on thin slices of fixed samples of mice liver that contains small tumor nodes initiated by intrasplenic injection of transformed rat liver cells. The design of the SAPT experiment in this case includes labeling of the tumor tissue by a fluorescent dye in order to be able to precisely find the place to perform the SAPT experiment. For this purpose, in addition to the standard tonometry equipment, we use a fluorescent microscope placed above the sample in order to monitor the exact region (tumoral or sane) exposed to the shot by the SAPT air jet.

The size of the cancerous cell clusters formed in the mice liver is within the limits of $80-100 \mu \mathrm{m}$, while the spatial resolution of a local stress induced by the air stream delivered by the SAPT pipette is in the order of $\sim 60 \mu \mathrm{m}$. The deformation of the tissue can be measured with a vertical sensitivity of $\sim 1 \mu \mathrm{m}$. Figure 8(a) shows the general view of the liver, where the cancerous nodes are seen as the whitish regions. In Fig. 8(b), a single cancerous locus, which is fluorescent, is well seen.

In order to perform an unbiased comparison of compliance of sane and cancerous tissue, we performed several sets of SAPT experiments on cancerous nodes (fluorescent regions) and neighboring sane tissue (nonfluorescent areas). The distance between any sane and a cancerous region under paired comparison does not exceed $100 \mu \mathrm{m}$. This way, we take into account the initial histological variability of the liver tissue and its further complexity upon formation of cancerous nodes.

Figure 8(c) depicts the deflection of the light spot position of a single tumor-sane region pair. Figure 8(d) summarizes the results of several SAPT experiments, where each data point shows the value of the maximal deflection of the light spot during the SAPT experiment. The set of data obtained shows a systematic higher deformability of the sample in the sane areas as compared to the cancerous ones. Hence, even in fixed tissue, the immature tumorous regions are stiffer than the healthy regions.

\section{Case study 4: Limb buds}

An important question in developmental biology is that of the location of the point of outgrowth of limbs [29]. A recent 

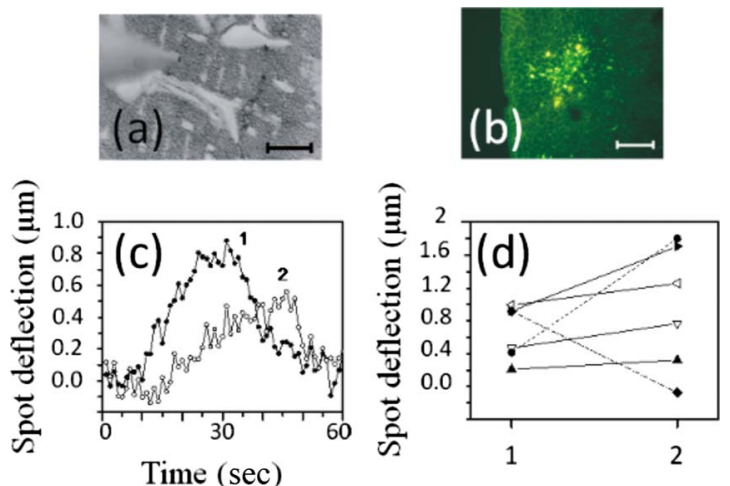

FIG. 8. (Color online) Comparison of compliance of cancerous and healthy tissues on an example of liver tumor in mice. (a) Direct optical image of the liver sample, where cancerous nodes appear as the white zones, the tip of the SAPT pipette is seen on the left, and the size of the bar is $300 \mu \mathrm{m}$. (b) Magnified view under a fluorescent microscope. The large voids are sections across blood vessels. The scale bar is $50 \mu \mathrm{m}$. (c) Tumor compliance (curve 2) and sane tissue compliance (curve 1) in two regions very close to each other $(<100 \mu \mathrm{m})$, as assessed by the SAPT. Please note that the sample is fixed. During the first seconds of the air puff, a very large compliance difference is found which progressively reduces. This reduction in compliance contrast is ascribed to desiccation. (d) A set of data shows an almost systematic higher deformability of the sample in the sane (1) area close to a given cancerous (2) area. The distance between the places where the SAPT experiments are performed is $<100 \mu \mathrm{m}$ in each pair. One point was found to be "wrong way."

mathematical analysis of the problem of gastrulation motion shows that a hyperbolic flow of cells suffices to localize the limb lateral plates from which the limbs emanate in the shape of four domains located two above the navel and two below [30-32], Fig. 9. This explanation ascribes the forma-

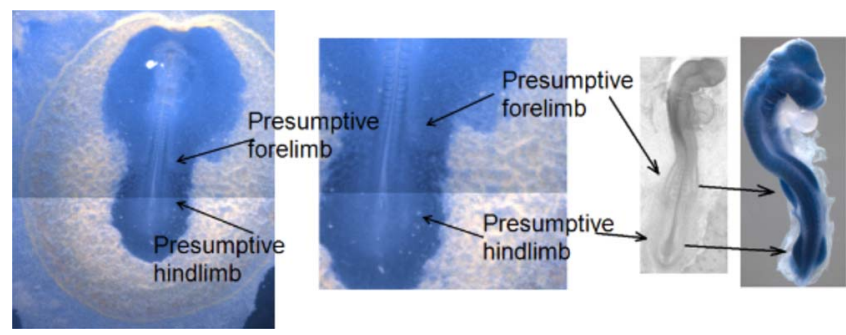

FIG. 9. (Color online) (Left) An early embryo (day 2) at the stage where lateral plates form only small bumps. These bumps find themselves on each side (anterior and posterior) of the region of the presumptive navel (direct transmission illumination of a rinsed embryo). Already at day 2, the presumptive limb areas are visible in the shape of small round thickenings formed by the engulfment of the ectoderm, which rotates toward the posterior part and toward the anterior part, thus creating the limb fields. (Right) Later stages of embryo development, showing how the buds actually emanate from the initial engulfment (from database Geisha), which outgrows progressively up to the point where the buds become true limb paddles. The tail bud grows along the anteroposterior axis, passing over the limb plates, and generating the well-known rotational shape of the pelvis. tion of paired appendages to the topology of the initial flow, which scales up dynamically the areas of the limbs, rather than to two discrete, ad hoc "inductions" occurring along a gradient of chemicals acting as local "morphogenes."

In the early blastula, prior to formation of the body axis, there exist vortex flows [31-35] (Fig. 10). The flow pattern is convergent toward the presumptive navel and divergent away from it, as actually observed and confirmed by numerous studies, such that progressively the cellular sheets form bumps in the areas of the presumptive hips and shoulders $[15,33]$ defined by the early cellular distribution. These domains will grow out the limb buds, in the form of the wellknown little limb paddles. This model, based on the actual cell tracts, is at variance with existing "induction" models of development [36,37]. In particular, due to vorticity, the flow exhibits cell tracks oriented in opposite directions in the same field, which is incompatible with chemotactic models. One prediction of the physical model ascribing limb morphogenesis to the tissue flow is that the limb bud areas from which the limb buds emanate should be low-pressure regions, associated to tissue engulfment (rotation and translation toward the median axis), as compared to the rest of the body in between limbs, which is more compressed. This is due to the following fact.

Direct inspection of limb buds in embryos shows that they correlate with the winding of the tissue. Now, careful analysis of the tissue flow pattern in early embryos shows that there exists indeed a stagnation point $[31,32]$ such that the flow revolves away from the stagnation point. Cells converge toward the stagnation point in the left-right direction and diverge from the stagnation point in the anteroposterior direction. This remarkable situation can be analyzed at first order in terms of a hyperbolic flow [32]. In effect, the presence of a stagnation point $(V x=0, V y=0$ at $x=0, y=0)$ allows one to perform a linear expansion of the flow around the stagnation point [Figs. 10(a) and 10(b)]. It is well known that such a linearized flow writes $(V x, V y)=(-k x, k y)$, which satisfies the mass conservation law $\operatorname{div}(V)=0$, around a singular hyperbolic point. If we restrict ourselves to this linearization, we see that such a flow derives from a viscous flow pushed by an in-plane pressure field which has the form $P(x, y)$ $\sim x^{2}-y^{2}$. This is to say that the pressure will be high along the flanks and toward the presumptive navel area (stagnation point) and will decrease toward the limb plates, following the advection of the tissue downstream.

We therefore expect the point of outgrowth of the limbs to correlate with a low-pressure area, from which further growth will be easier. More specifically, submitted to a mesodermal expansion, the existence of an in-plane stress higher in the region of the stagnation point and lower away toward the revolving cores of the vortices will imply a localized bulging of the limb plates by out-of-plane equilibrium. In addition, although not the core of our study, epigenetic transduction effects of the pressure gradient are likely to be involved.

In order to confirm our prediction that the presumptive limbs areas have a lower stress state, we have analyzed by tonometry the presumptive limb bud areas [Fig. 10(f)]. A direct visual inspection of the air jet impact (data not shown) shows that the limb bud area is much softer than the more 
(a)

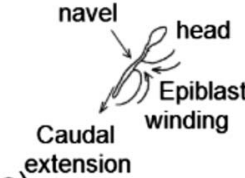

(a)
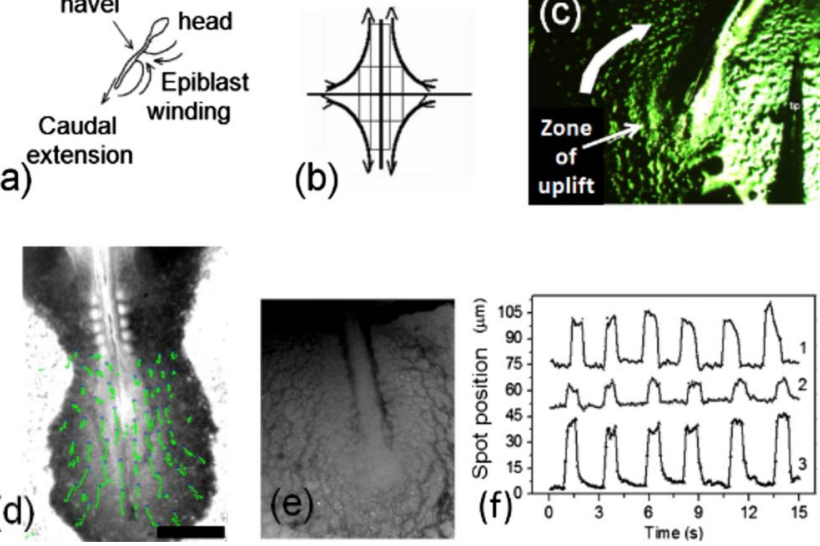

FIG. 10. (Color online) (a) The engulfment of the ectoderm and the elongation of the $A-P$ axis is associated to a dynamic rotation of the tissue and hence to a stress which decreases from the presumptive navel area and toward the shoulder and hips regions, which will physically be low stress areas. (b) The early linearized flow is hyperbolic around a stagnation point which is the presumptive navel. (c) Shadowgraph inspection of the early embryo shows the trails of the epiblast engulfment and that the uplift of the epiblast colocalizes with the zone of winding. (d) The cell paths exhibit a vortex dynamics, as they approach the center of the body, prior to rotate caudally (the green lines correspond to time-lapse tracking of cell paths during $5 \mathrm{~h}$, with $5 \mathrm{~min}$ interval, on a chicken embryo; the starting point of each track is on the blue point). Scale bar is $500 \mu \mathrm{m}$. (e) The cell dynamics has many anatomic consequences, for example, eventually, the tissue winding is directly visible in the path taken by the haemagioblasts which form the capillary lattice (visible here as a black mesh). (f) Measurement of the stress. We analyzed by SAPT the area of the presumptive limb buds and found that limb buds are much softer than the body. The plate in (f) shows the deformability in the forelimb plate (top, data 1), in the flanks in between (middle data 2), and in the hindlimb plate (bottom data 3). Data represent the spot position as a function of time for a series of six rapid air puffs exerted in situ. The limb plates show a much larger deformability (more flexible area). The outgrowth of limbs is quite clearly related to a higher deformability of the limb plates at early stages of development. Limb outgrowth is therefore correlated with the stress gradient and the corresponding deformation field, inherited from the early pattern of gastrulation movements.

proximal parts (flanks and dorsal areas) of the body. We oriented carefully the limb plates so that a similar spot was obtained proximally (on the back) and distally (on the lateral plate or bud) and analyzed the amplitude of the deformation, at very early stages, when limbs are absent and only identified by a modest bump or bud. The measurement by the spot shows a large compliance difference between the lateral plates and the more proximal parts and along the flanks. Figure 10(f) shows deformability data in the presumptive hindlimb, forelimb, and flank areas. This shows that the region of the presumptive limb paddle is much softer than the presumptive flanks of the body. This explains naturally the direction of further growth: after localization by the tissue winding, physical growth of limbs will next be oriented in the direction of decreasing stress by Newton's law. Of course, it may also be possible that there exists a difference in material property, in addition to the stress state. However, the flow pattern implies necessarily a stress gradient, which is in itself sufficient to explain the physical drift and final localization of the soft areas, and further epigenetic differentiation of cells. Assuming that there exists a priori an ad hoc softer area (e.g., lower Young modulus), by some chemical coincidence or "induction," implies an additional causal explanation of the localization, which is currently lacking, if it is not by the hyperbolic flow.

\section{DISCUSSION AND CONCLUSION}

The results reported in this paper cover the most important experiments performed by a unique self-developed instrument (high-resolution scanning air puff tonometer), which is capable of in vivo measuring of the stiffness tissue without any contact, if we consider the air jet as noninvasive. The first set of experiments presented here serves to test the capabilities of the device; another set is devoted to study the role of gradients of mechanical properties in the processes of tissue or cellular aggregates development.

The use of this new instrument shows that it is possible to locally indent with a rapid air jet biological surfaces. Since an air puff is used for the indentation, there is very little contact and the point under study can be shifted rapidly to some other place to analyze dot-by-dot gradients of material properties. It allows one to do spatially resolved analysis without dragging an instrument across the living sample. In existing techniques, such as optical tweezers [8] or contact cantilevers [9], it is not so easy to perform measurements in different parts of the same, fresh, sample. Acoustic elastography is a more promising technique in terms of imaging mechanical contrasts [12], but the spatial resolution is still low and the data need a nonintuitive interpretation in terms of acoustic waves. When operated skillfully, the SAPT allows one to gather instantaneously very valuable information on viscoelastic properties of materials. The acoustic tip described in Ref. [17] might help to improve both resolution and mechanical analysis.

Nevertheless, some drawbacks still exist. First of all, the method of data acquisition we use, which is based on optical detection, limits the possible shapes of the surfaces under study to the ones that are close to flat. However, as the instrument's resolution is quite high, due to the diameter of the air stream which can be diminished down to $10 \mu \mathrm{m}$, flexibility of the setup, which allows one making a precise adjustment of the air jet, sample, and the detector optics, the size of the flat area, which is required for proper measurements, is quite small. For example, we were able to compare the mechanical properties of chicken embryo skin in areas of developing feather buds to the flat skin areas in embryos Hamburger and Hamilton (HH) stage 35 and 37 as the diameter of the feather primordia in this stage is $150-200 \mu \mathrm{m}$, which is large enough for the measurements. The results are presented in a separate paper [38].

Moreover, as it is often not possible to expose the biological objects to the air puff for a long time due to the progressive desiccation of the samples, especially the softest ones, the instrument is only suitable to study the surfaces whose 
responses to the air puffs have relaxation times in the range of fraction of a second to minute. For the same reason, the number of experiments performed on the same object is limited by its rate of desiccation. At the same time, due to minimal contact of the instrument with the object under study, the damage of the tissue during the experiment is negligible, thus it is possible to perform the measurements in vivo and to repeat the experiment on the same object after a variable period of incubation. Reproducibility of the data is very good. In this way, monitoring of the changes in mechanical properties of the tissue due to some external or internal disturbances may be studied.

In this paper, we also present the results of the study of mechanical properties of different kinds of developing tissues which are done by the SAPT tonometer. In all systems studied, the instrument shows readily that there exist physical gradients at all scales, ranging from the entire organ shape (e.g., limb bud, flanks) to small details, such as the neighborhood of the smallest arteries. Such gradients have morphogenetic properties, which are complementary to direct genetic inductions, and rarely emphasized because they are so difficult to image spatially. It has been proven recently that biomolecular stainings in lung tissue may be correlated with strain maps [39]. In addition, the pattern of most genetic maps during early gastrulation can be analyzed as a simple consequence of tissue advection [32], therefore a possibility arises that many colored images provided by staining techniques be in fact impossible to interpret without a clear picture of the stress and deformation maps.

Possible developments of the instrument are (i) ultraresolutive tips, in the nanometer range, to turn the SAPT into an "air jet AFM," (ii) water puff tonometry to avoid dessication or to treat samples inside buffers such as phosphate buffer solution (PBS), (iii) Nomarsky mode tonometry, which allows one to measure the deformation with a higher accuracy, or, in other contexts, (iv) high-speed tonometry with rapid cameras to get a more resolved response curve of quite elastic materials. All these technical improvements will be progressively tested.

\section{SAMPLE PREPARATION}

\section{A. Bacteria colonies}

The Proteus mirabilis wild-type strain WT19 corresponds to the clinical isolate U6450 [40] from a chronic urinary tract infection (UTI) involving renal stone formation. WT19 was grown in lysogeny broth medium at $37^{\circ} \mathrm{C}$ overnight. For studying periodic swarming, a $3 \mu \mathrm{L}$ of culture broth was deposited at the center of a Petri dish and was allowed to grow for up to $18-24 \mathrm{~h}$ on the LB agar $2 \%$.

The fluorescent labeling of the colony is obtained by shedding a so-called dead-live solution onto the colony. The dead-live solution (Molecular Probes) contains SYTO9 and isopropidium. It is known that only the SYTO9 enters living cells which then fluoresce green. Dead cells or inert materials transfer both SYTO9 and isopropidium, in which case the fluorescence is red. The agar will appear dark in the confocal microscope and the colony brighter as observed.

\section{B. Tumor samples}

The cancer data were prepared in the following way. Transformed rat liver epithelial cells (F1) were labeled with the lipophilic tracer Dil (molecular probes). The fluorescent cancer cells were injected directly intraspleenic. The cancer nodule was allowed to develop in vivo for 6 days, after which the mouse was sacrificed. Histological slices of the liver (40 $\mu \mathrm{m}$ thick) were cut and fixed with paraformaldhehyde $4 \%$. The precocious tumor tissue is identified with classical fluorescent microscopy (Leica microscope MZFLIII). The cancerous cell clusters are $\sim 80-100 \mu \mathrm{m}$ big.

\section{Chicken samples}

Pathogen-free, fertilized chicken eggs (Gallus gallus and white leghorn eggs) were incubated at $37.5^{\circ} \mathrm{C}$ in a humidified incubator and embryos were staged according to $\mathrm{HH}$ [41].

\section{Blood vessels}

For the blood vessel measurements, the chick embryos aged between 35 and $60 \mathrm{~h}$ were used for the yolk-sac experiment and the chick embryo aged between 2 and 4 days were used for the Formation of vitelline arteries and veins experiment. The albumen was drained out from a hole in the blunt end of the egg's shell then a square window was opened in the shell. After cutting out the membrane surrounding the chick embryo's yolk sac, the embryo was rescued and washed in a PBS. The embryo was spread on a lamella and the excess of PBS was removed by capillarity with a sterilized paper until the yolk sac appeared lying flat on the lamella, with no yolk or albumen remaining underneath. Samples were transferred either to the microscope for optical imaging or to the tonometer for tonometry analysis and studied within $30 \mathrm{~min}$. A typical tonometry data acquisition takes less than $1 \mathrm{~min}$.

The in vivo time-lapse images were performed inside a minitüb standard incubator with a glass window and under a Leica MZFLIII stereo microscope; illumination was by a Shott fiber lamp with halogen dichroic light (24V, 250W). In order to avoid condensation on the window, the embryo is placed inside a double chamber with a warmer side on top $\left(38.7^{\circ} \mathrm{C}\right)$ as compared to the bottom plate $\left(37^{\circ} \mathrm{C}\right)$.

\section{Limb buds}

For the limb bud measurements, two types of experiments were performed. Early embryos HH stages 11-13 were studied in vivo, in ovo. Older embryos from $\mathrm{HH} 13$ to $\mathrm{HH}-16$ were extracted from the eggs. The yolk was carefully rinsed with the PBS solution. The embryos were spread out on the Petri dish. The measurements were done within the $30 \mathrm{~min}$ time interval after the extraction in order to prevent measurement artifacts due to the sample desiccation. Limb buds are generally thought to appear by stage 17 of Hamilton and Hamburger classification. Our observations show that in fact, the areas of limb buds are committed to form limbs much earlier by the winding of the tissue. The uplift of limb bud 
areas is a dynamical process, not a stop-and-go discrete induction.

\section{ACKNOWLEDGMENTS}

This work was supported by a grant from CNRS in the STIC-SANTE program, EMBO short-term young scientist foundation (Contracts No. ASTF 60.00-06 and No. ASTF 200.00-07), and by a grant for foreign post-docs of the French Cancer Research Association (ARC). We thank Sylvie Lorthois for interesting discussions and especially for providing the Ref. [16] and we thank an anonymous reviewer for constructive comments and for providing Ref. [18].

\section{APPENDIX: CALIBRATION EXAMPLES}

We present in this appendix physical measurements and controls which serve to understand how the tonometer functions and what is measured.

\section{Tip orientation}

Figure 11 shows an air puff experiment on distilled water. The same experiment is shown for the best pipette orientation and for an orientation rotated by $180^{\circ}$ of the pipette. This clearly illustrates the importance of the pipette orientation. However, once the calibration is done, even for a poor signal, an absolute calibration of deformation vs force is achieved. In Fig. 11 (left), the observed slope of the response of the water surface deformation during the air puff is linked to the viscoelastic (oscillatory) damping.

\section{Absolute calibration}

A material of known physical properties can be used for absolute calibration. Figure 12 shows the same experiment performed on distilled water and on ethanol.
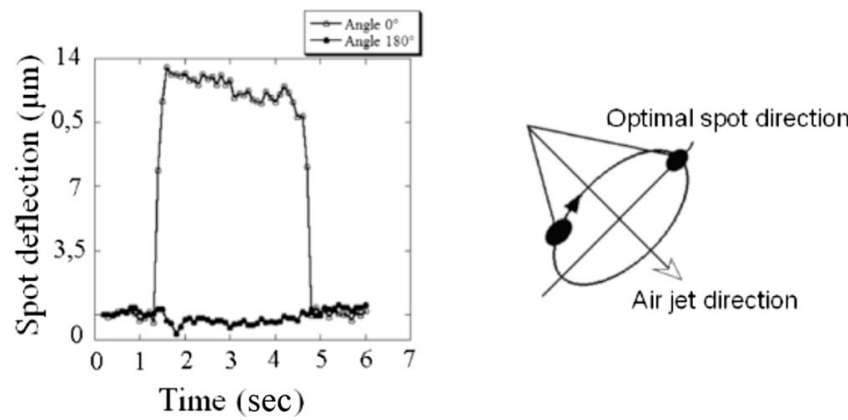

FIG. 11. Comparison of water data to the same tip, for a reference angle noted $0^{\circ}$, and with a rotation of $180^{\circ}$ around the tip axis. The positive displacement (large) becomes a negative displacement (very small). The pipette angle must therefore be carefully adjusted. This is why the rotation is motorized around the tip axis, to optimize the angle, when installing a new tip. The sketch to the right explains the angle which is rotated in order to achieve the optimal spot direction. The spot light direction is rotated until it finds itself slightly "ahead" of the air jet.

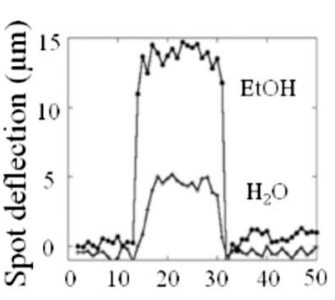

Time $(0.1 \mathrm{sec})$

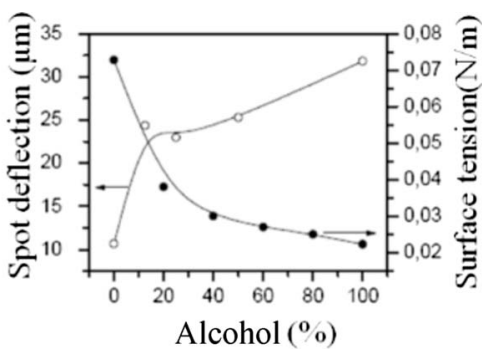

FIG. 12. (Left) Typical measurements on pure water (distilled) and absolute ethanol. (Right) Spot deflection as a function of ethanol concentration in distilled water. In S2-right, the black dots represent the alcohol-water surface tension and the circles the measured spot deflection. The surface tension decreases with increasing ethanol concentration, hence the deformation increases.

\section{Distance calibration}

The deformation imparted on the surface depends on the exact tip-to-surface distance because of progressive viscous diffusion of the air jet into the steady air surrounding the tip. Therefore, the indentation is sharper at closer distance. However, another effect exists: when the air jet and light spot are not strictly coaxial (which is generally the case), there exists a mismatch between the actual area of air impact and the actual illuminated zone. This mismatch increases for higher tip-to-surface distance. When the air jet is quite coaxial with the light, the spot deforms in an annular fashion; this sort of deformation has a deflection component in both directions, therefore reducing the deflection component which is measured. Therefore, the detection may vary nonlinearly with the distance. Figure 13 shows a typical curve of deflection as a function of tip-to-surface distance taken on water.
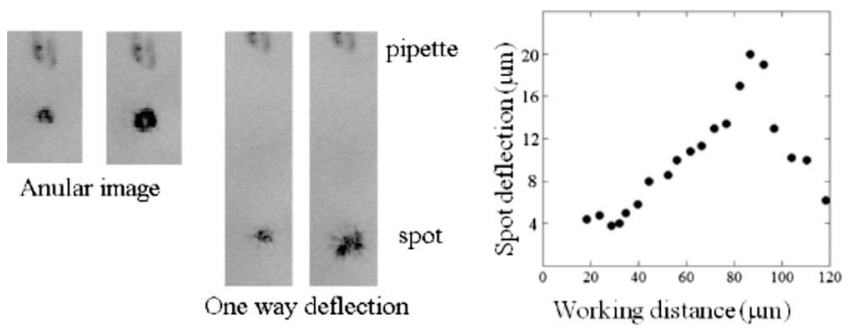

FIG. 13. Deformation as a function of distance between the tip of the pipette and the sample surface (for the same pressure 11 millibar). With a short distance, as shown on the image to the left, the spot has somewhat of an annular deflection image inside the deformation cuvette. With a longer distance, the spot is more offset with respect to the cuvette and it has a truly one way deflection. The deflection first increases and then decreases as a function of tip-tosurface distance. This effect is easily understood in the following way. When the tip is very close to the surface, the spot is more centered around the air puff, therefore the spot deflection has a component in both directions (since the deformed spot has an annular shape). When the tip-to surface distance is increased, the light spot and air puff are more offset. The deflection becomes more one way because the light spot is on one slope of the cuvette only. However, as the tip-to-surface distance increases, air diffusion decreases the air impact, therefore eventually decreasing the force at the impact area. 


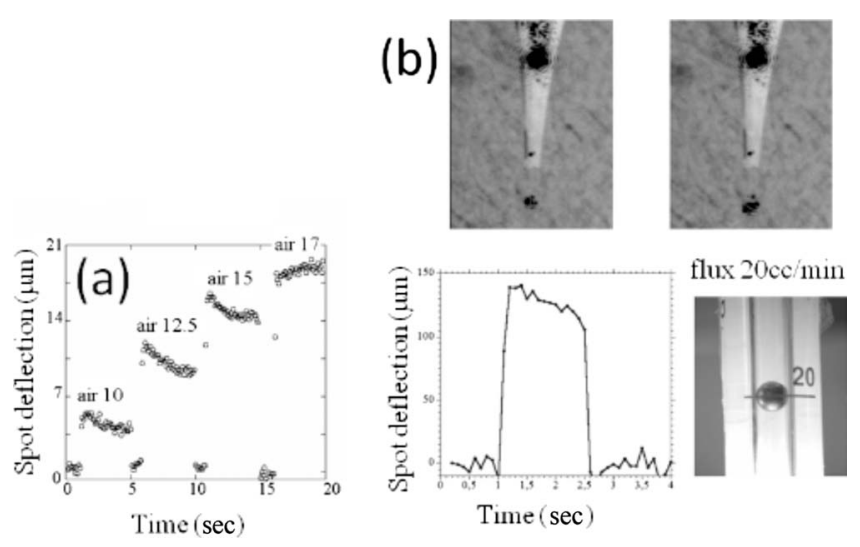

FIG. 14. (a) Deformation as a function of the applied air pressure at the entry of the line (in millibar over atmospheric pressure). The initial overshoot is a real effect. When a surface (here of water) receives the air puff, there is an initial elastic overshoot, which is rapidly damped. However, for stronger puffs, this effect disappears and the convergence to the limiting value is overdamped. We ascribe this to the tension imparted on the surface by the air puff: for a higher tension, the surface is stiffer and hence oscillates less. However, in the range of parameters studied, the deformation is linear with force, hence allowing direct interpretation of data. (b) Large absolute deformation of a water surface $(130 \mu \mathrm{m})$ at a large absolute flux $(20 \mathrm{cc} / \mathrm{min})$ for a pipette which is $110 \mu \mathrm{m}$ in diameter. The top images show the air off-air on spots. The bottom images show the actual spot displacement detection and the fluxmeter bead. The corresponding air flow of density $1.2 \mathrm{~kg} \mathrm{~m}^{-3}$ is approximately $3 \mathrm{~m} / \mathrm{s}$ and hence the corresponding force of the impinging air flux is approximately $F=10^{-6} \mathrm{~N}(F$ $=\left[3 \mathrm{~ms}^{-1}\right]\left[1.2 \mathrm{~kg} \mathrm{~m}^{-3}\right][20 \mathrm{cc} / \mathrm{min}] \sim 3 \times 1 \mathrm{~kg} \times 20.10^{-6} / 60 \mathrm{~N}$ $\left.\sim 10^{-6} \mathrm{~N}\right)$.

\section{Pressure calibration}

Of course, since we expect the surface deformation to vary with material properties, for a constant force, we also expect the same material to have an increased deformation for increasing values of the impacting air puff. Figure 14(a) shows the deflection measured on distilled water for different pressures measured at the entry of the air line. The overshoot which is seen at the beginning of the air puff is a real effect.

The air flow was also measured in parallel with a floating bead flux meter (Meterate). For example, Fig. 14(b) shows the tip, the deformation, and the air flux, as measured in the same experiment. In this case, the force exerted is approximately $10^{-6} \mathrm{~N}$; for biological samples, the force was more in the $10^{-8} \mathrm{~N}$ range. However, we found that the pressure indication was more reliable, as the floating bead tends spuriously to be arrested at aberrant positions due to friction along its glass tube.

The deformation of the surface is also dependent on the geometrical boundary conditions. Indeed, in principle, the deformation of a flat membrane is related to its tension and also to its diameter. This is why all calibrations examples and material comparisons (water or alcohol) are made in Petri dishes of exactly the same size. In vivo, it is clear that the gross geometry of the tissue may come into play for the determination of the deformability. However, this is consis-
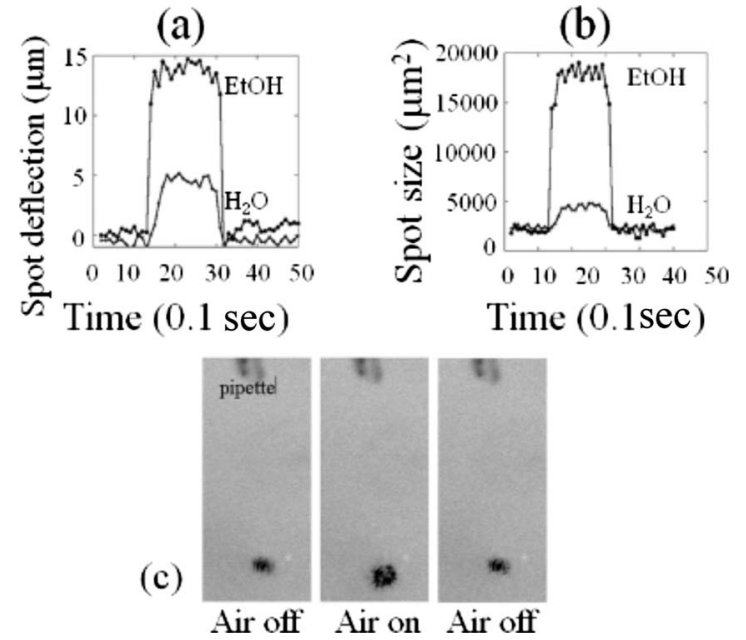

FIG. 15. [(a) and (b)] Comparison of a typical spot deflection and spot size data for water and ethanol, acquired simultaneously. (c) When the air puff and the light spot are close to coaxial, the air spot undergoes a dilation (magnification) in addition to a deflection. Then, both the size of the spot and the position of the spot can be followed to gather information about the surface deformation (in this experiment, the spot size is $50 \mu \mathrm{m}$ ).

tent with the morphogenetic process: when a clutch of cells push themselves forward, what matters is the neat deformation which is obtained, for a given force, including any possible boundary effects. In this respect, the push action of the air puff is conceptually analogous to the push action of cells.

\section{Spot size mode}

When the spot size is very well centered in the deformation cuvette, the image of the spot is almost centrosymmetric and therefore the deflection of the center of gravity of the spot is small. However, in this case, the radial variation of the spot size amounts to an increase of the surface area of the

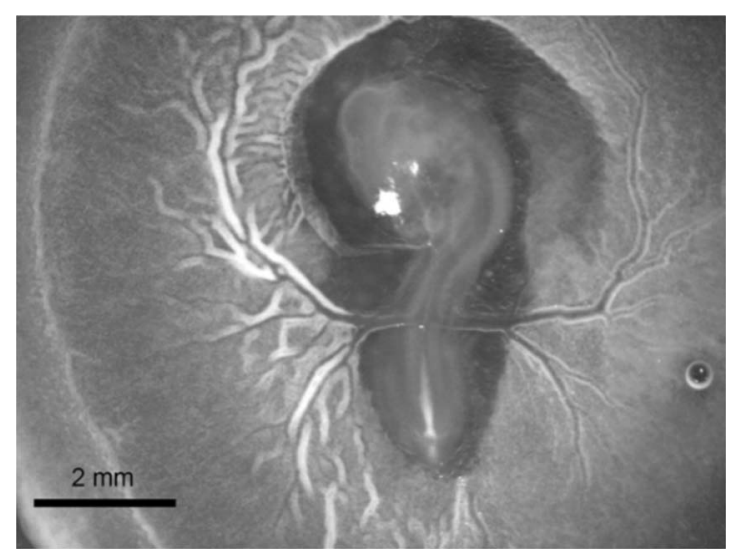

FIG. 16. Chicken embryo as observed in direct illumination. Right side shows normal vessels, while left side shows a very conspicuous swollen area along the arterioles. This effect is obtained when culturing embryos shell-less in a Petri dish. Simple PBS buffer is added on top of the left half and normal yolk on top of the control (right) half. 
spot on the detection charge coupled device (CCD) of the camera. In this situation, one can switch the tonometer to a "spot size" mode. Figure 15 shows two examples of water $\left(\mathrm{H}_{2} \mathrm{O}\right)$ and ethanol $(\mathrm{EtOH})$ data, observed simultaneously in spot deflection mode (a) and spot size mode (b). Figure 15(c) shows the actual spots during the ethanol data acquisition. The deflection follows the center of gravity of the spot and the spot size follows the surface area of the spot. Data show that both modes can be accurately followed; the size mode may help to confirm the data in deflection mode (this is analogous to the so-called $A-B$ and $A+B$ signals of the atomic force microscope). The spot size mode is less sensitive to noise than the spot deflection mode, however, it may be sensitive to surface reflectivity. From a geometrical point of view, the spot size mode gives the size of the blurred image of the spot across the equivalent diopter of the deformed surface. In the small deformation regime, this amounts to a magnification, which is proportional to focal length.

\section{Appendix Part 6}

Cell swelling in the yolk sac is not directly related to the uptake of fatty acids from the yolk itself toward the endoder- mal cells of the yolk sac. Indeed, we performed an assay in which the embryo (aged 3 days) was turned upside down in a Petri dish. The yolk was rinsed out carefully (5 samples). The embryo lies in the center of the image, the anteroposterior axis being vertical. The yolk sac is visible in the background and the right and left vitelline arterial trees are very clearly visible (except that they are inverted with respect to the usual observation, since the embryo and the entire yolk sac were turned upside down). The embryo is alive. At time $T=0$, few drops of yolk are put back on one half of the embryo and the embryo is put back to incubate. The picture is taken $10 \mathrm{~h}$ after adding the yolk to the right half. The embryo is nourished from one side of the yolk sac and the evolution of the vessels can be followed directly. The result, shown in Fig. 16, reveals that cells along the vessels located on the side devoid of yolk (left half in Fig. 16) dilate more than the ones on the contralateral side exposed to yolk (serving as control). This is quite conspicuous in the form of the whiter and thicker area along the vessels (compare to Fig. 7 of the main text). In areas where inflated cells of the bank of one vessel merge with the ones of the neighboring vessel, the two vessels become sharply parallel.
[1] L. V. Beloussov and R. Gordon, Int. J. Dev. Biol. 50, 79 (2006).

[2] N. Tsikolia, Int. J. Dev. Biol. 50, 333 (2006).

[3] L. V. Beloussov, The Dynamic Architecture of Developing Organism (Kluwer Academic Publishers, Dordrecht, 1998).

[4] D. E. Ingber, Int. J. Dev. Biol. 50, 255 (2006).

[5] V. Fleury and T. Watanabe, C. R. Biol. 327, 663 (2004).

[6] A. M. Cheshire, B. E. Kerman, W. R. Zipfel, A. A. Spector, and D. J. Andrew, Dev. Dyn. 237, 2874 (2008).

[7] T. C. Skalak and R. J. Price, Microcirculation (Philadelphia) 3, 143 (1996)

[8] K. C. Neuman and S. M. Block, Rev. Sci. Instrum. 75, 2787 (2004).

[9] S. M. Lindsay, The Scanning Probe Microscope in Biology in Scanning Tunneling Microscopy and Related Techniques, edited by D. Bonnell (Wiley, New York, 2004).

[10] M. Keller, J. Schilling, and E. Sackmann, Rev. Sci. Instrum. 72, 3626, (2001).

[11] Y. Tseng, T. Kole, and D. Wirtz, Biophys. J. 83, 3162 (2002).

[12] J. M. Schmitt, Opt. Express 3, 199 (1998), and references therein.

[13] A. A. Spector, W. E. Brownell, and A. S. Popel, J. Acoust. Soc. Am. 103, 1001 (1998).

[14] J. Nissl, Tonometry entry online medical portal: http:// www.healthbanks.com/PatientPortal/Public (2007).

[15] V. Fleury, A. Al-Kilani, M. Unbekandt, and T.-H. Nguyen, Organogenesis 3(1), 49 (2007).

[16] T. L. Labus and C. Aydelott, NASA Tech. Note No. D6368, 1971 (unpublished).

[17] J. Salençon, Visco-elasticité (Lavoisier, Paris, 1983).

[18] L. Brundin and I. Russell, Hear. Res. 73, 35 (1994).

[19] O. Rauprich, M. Matsushita, C. J. Weijer, F. Siegert, S. E.
Esipov, and J. A. Shapiro, J. Bacteriol. 178, 6525 (1996).

[20] E. Lahaye, T. Aubry, N. Kervarec, P. Douzenel, and O. Sire, Biomacromolecules 8(4), 1053 (2007).

[21] E. Lahaye, T. Aubry, V. Fleury, and O. Sire, Biomacromolecules 8(4), 1218 (2007).

[22] A. L. Romanoff, The Avian Embryo (Macmillan and Co., New York, 1960).

[23] R. Thoma, Untersuchunung über die Histogenese und Histomechanik des Gefässystems (Ferdinand Hencke Verlag, Stüttgart, 1893).

[24] T.-H. Nguyen, A. Eichmann, F. le Noble, and V. Fleury, Phys. Rev. E 73, 061907 (2006).

[25] A. Al-Kilani, S. Lorthois, T.-H. Nguyen, F. le Noble, A. Cornelissen, M. Unbekandt, O. Boryskina, L. Leroy, and V. Fleury, Phys. Rev. E 77, 051912 (2008).

[26] R. P. Rouhl, R. J. van Oostenbrugge, I. L. Knottnerus, J. E. Staals, and J. Lodder, J. Neurol. 255, 692 (2008).

[27] D. J. Padilla, P. McDonough, B. J. Behnke, Y. Kano, K. S. Hageman, T. I. Musch, and D. C. Poole, Am. J. Physiol. Heart Circ. Physiol. 291, H2439 (2006).

[28] J. F. Greenleaf, M. Fatemi, and M. Insana, Annu. Rev. Biomed. Eng. 5, 7 (2003).

[29] E. Mooney, Lower, eMedicine-Lower Limb Embryology Article, June 2006, http://www.emedicine.com/plastic/ topic215.htm

[30] V. Fleury, Organogenesis 2(1), 6 (2005).

[31] V. Fleury, Rev. Quest. Sci. 177, 235 (2006).

[32] V. Fleury, Eur. Phys. J.: Appl. Phys. 45, 30101 (2009).

[33] M. Callebaut, E. Van Nueten, H. Bortier, and F. Harrisson, J. Morphol. 255, 315 (2003).

[34] M. Chuai, W. Zeng, X. Yang, V. Boychenko, J. A. Glazier, and C. J. Weijer, Dev. Biol. 296, 137 (2006). 
[35] C. Cui, X. Yang, M. Chuai, J. A. Glazier and C. J. Weijer, Dev. Biol. 284, 37 (2005).

[36] R. L. Johnson and C. J. Tabin, Cell 90, 979 (1997).

[37] M. J. Cohn, J. C. Izpisúa-Belmonte, H. Abud, J. K. Heath, and C. Tickle, Cell 80, 739 (1995).

[38] O. P. Boryskina, Y. Le Grand, C. Legrand, and V. Fleury, Pro- ceedings of the European Microwave Association 2008, Vol. 4, p. 255.

[39] M. Unbekandt, P. del Moral, F. Sala, S. Belluschi, D. Warburton, and V. Fleury, Mech. Dev. 125, 314 (2008).

[40] C. Allison and C. Hughes, Sci. Prog. 75, 403 (1991).

[41] V. Hamburger and H. L. Hamilton, J. Morphol. 88, 49 (1951). 